\title{
Searches for new physics with displaced vertex signatures at the ATLAS experiment in LHC Run 1
}

9 January 2016 - Epiphany2016

Heather Russell, on behalf of the ATLAS collaboration
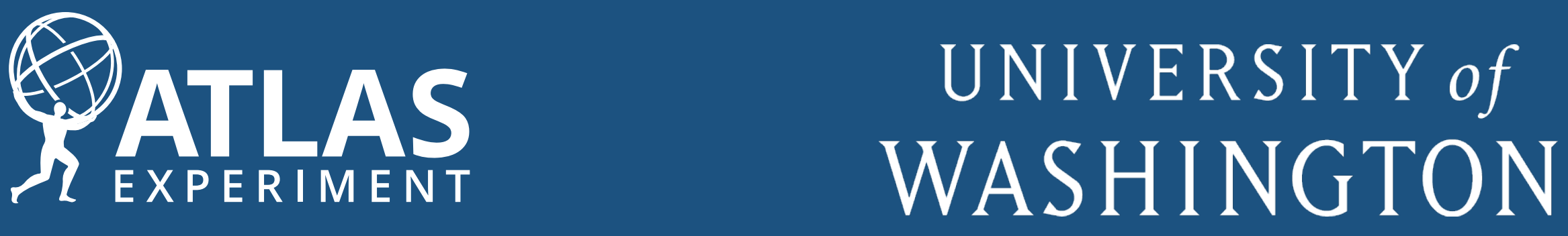
A few hints of new physics here and there... but where are the undiscovered particles hiding???

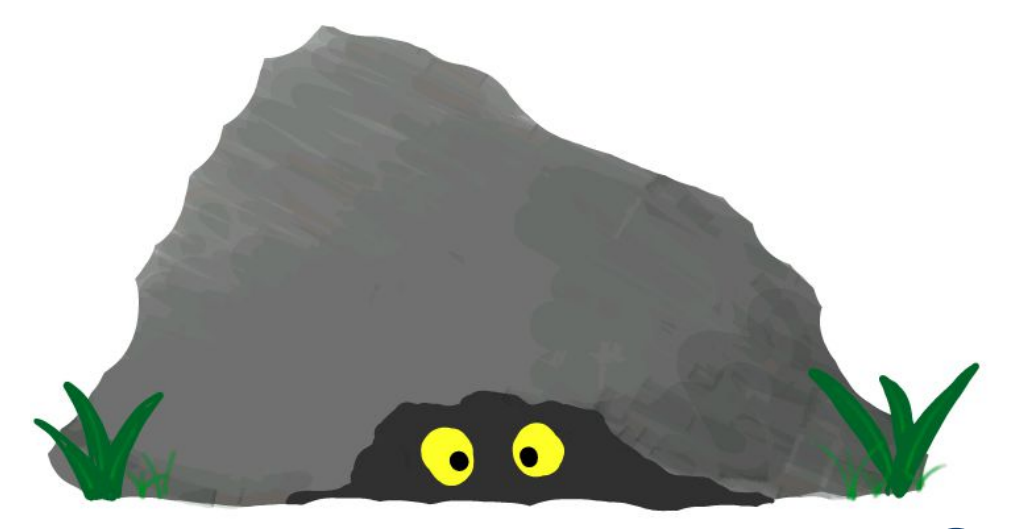




\section{Perhaps the new physics is not prompt:}
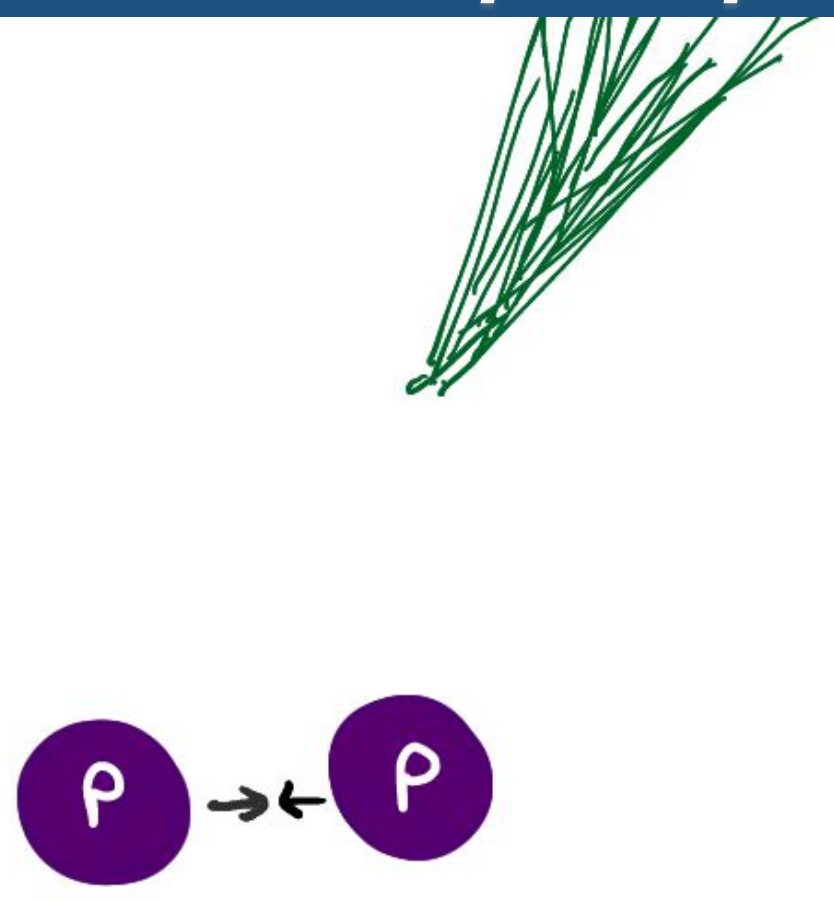


\section{what are displaced vertices?}

neutral, long-lived particles

decay displaced from the interaction point

leaving a displaced vertex signature 


\section{what are displaced vertices?}

neutral, long-lived particles

decay displaced from the interaction point

leaving a displaced vertex signature

On ATLAS, we can reconstruct displaced decays in the:

\section{Inner detector (ID)}

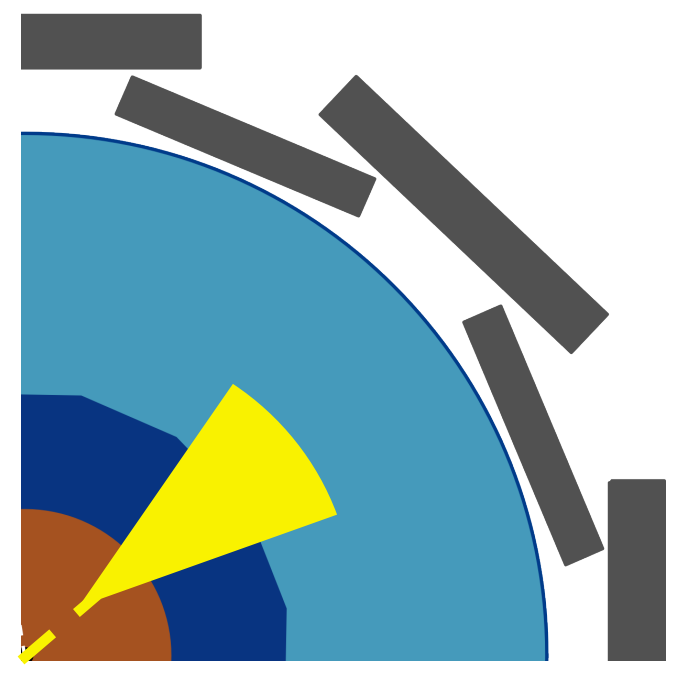




\section{what are displaced vertices?}

neutral, long-lived particles

decay displaced from the interaction point

leaving a displaced vertex signature

On ATLAS, we can reconstruct displaced decays in the:

Inner detector (ID)

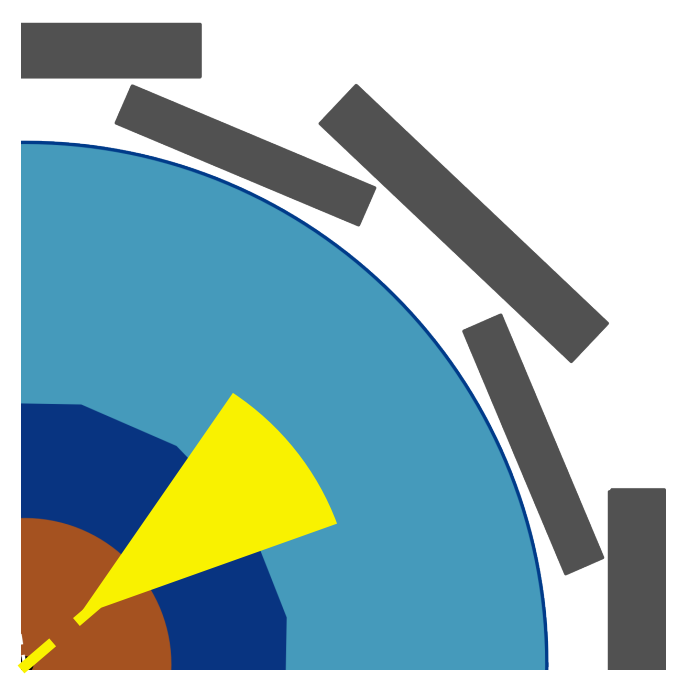

Heather Russell, University of Washington

\section{Hadronic calorimeter}

(Hcal)

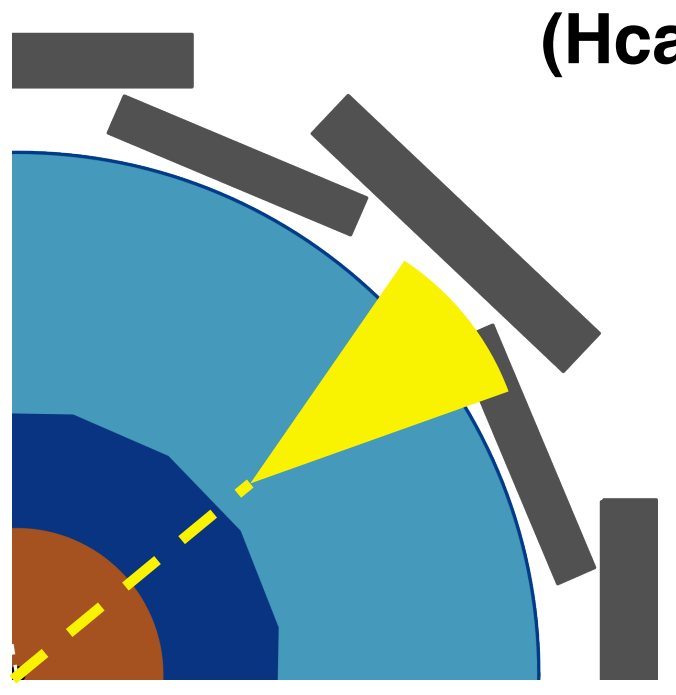

9 January 2016 


\section{what are displaced vertices?}

neutral, long-lived particles

decay displaced from the interaction point

leaving a displaced vertex signature

On ATLAS, we can reconstruct displaced decays in the:

Inner detector (ID)

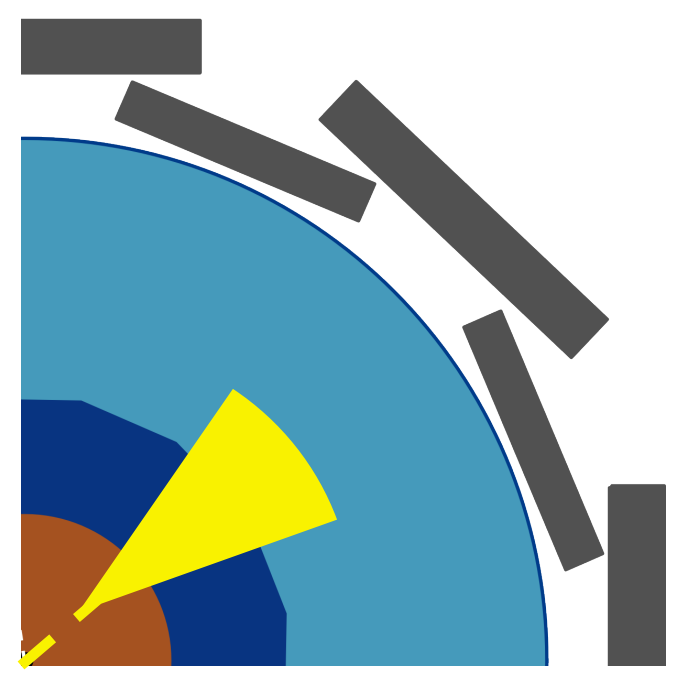

Hadronic calorimeter

(Hcal)

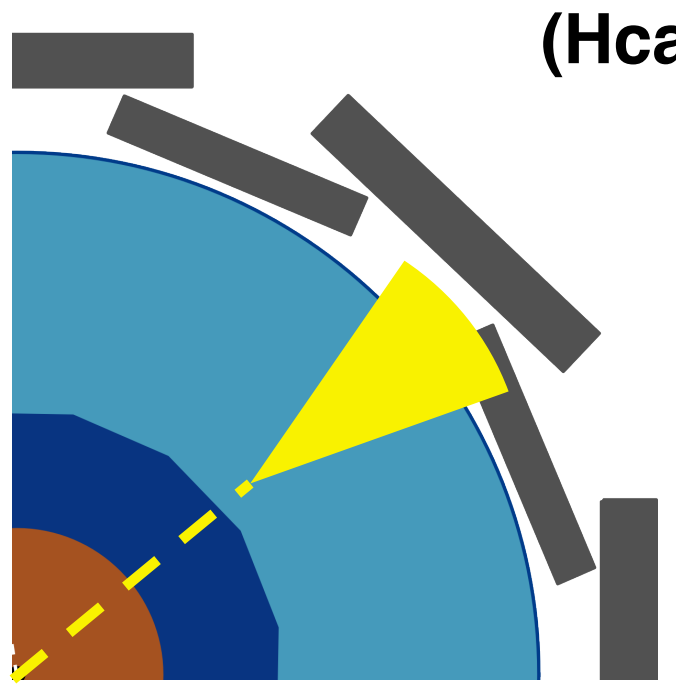

Muon spectrometer

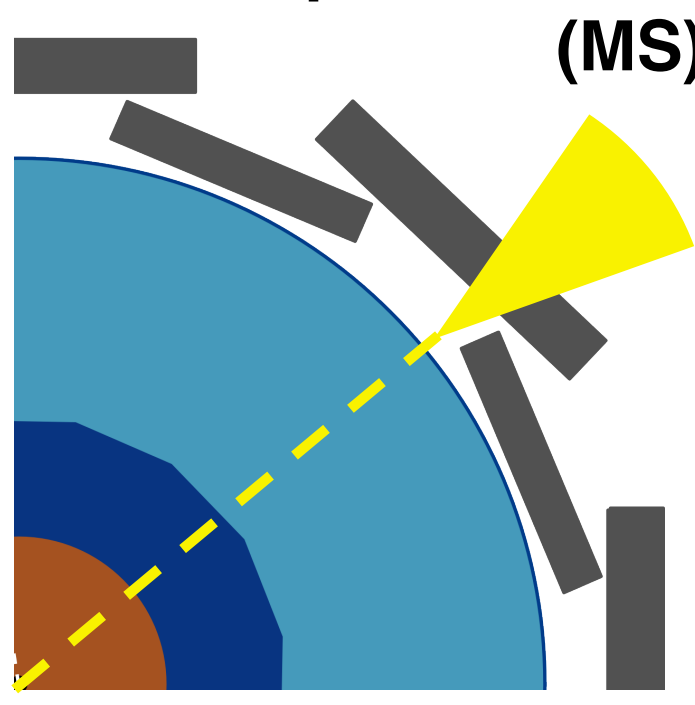




\section{Detection and reconstruction of displaced decays}




\section{inner detector vertex reconstruction}

1. ATLAS default tracking algorithm only reconstructs tracks with $d_{0}<10 \mathrm{~mm}$ $\rightarrow$ Modify to allow for high-d tracks

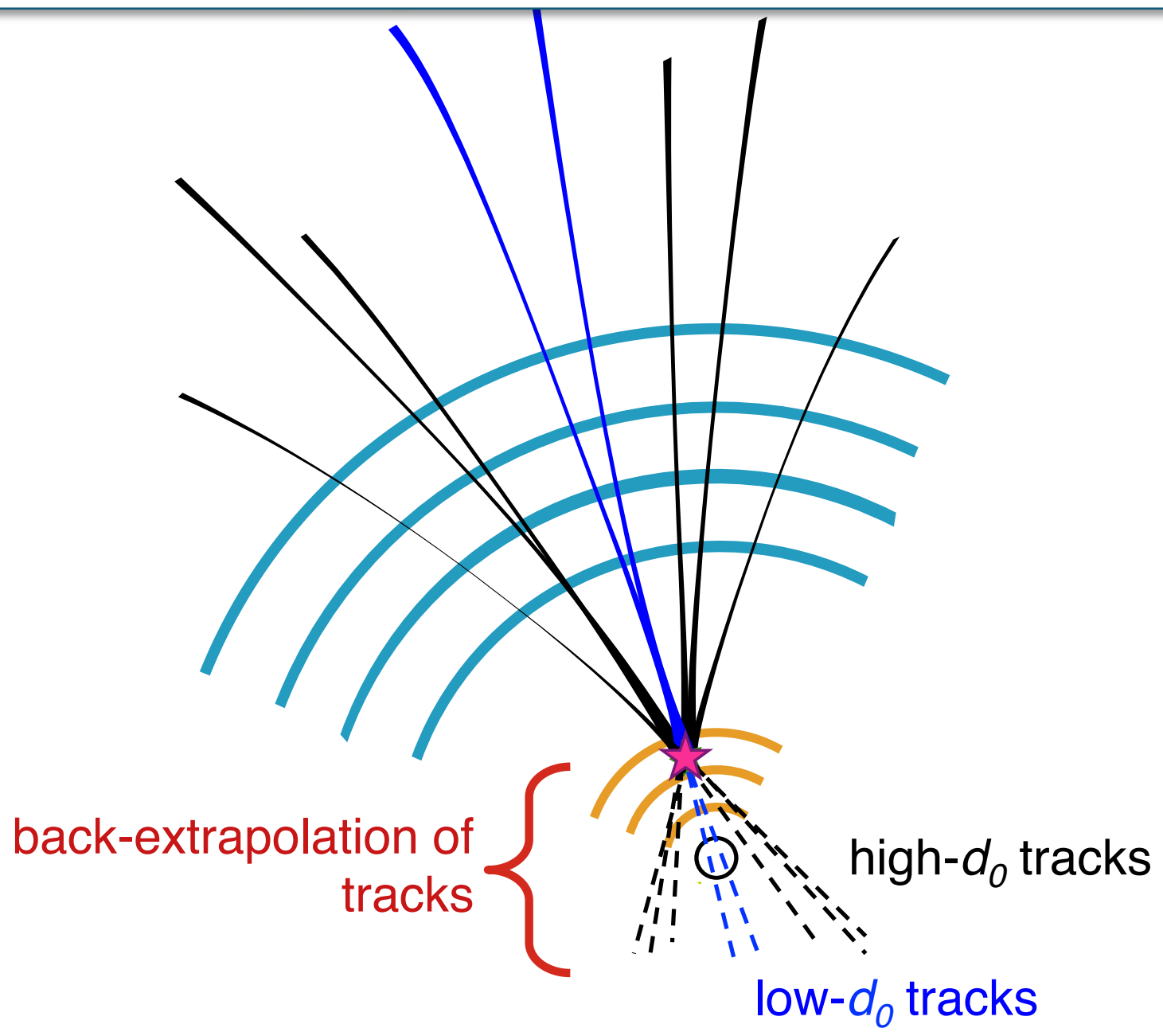

Plots: Phys. Rev. D92, 072004 (2015), arXiv:1504.05162

$2^{\text {nd }}$ IDVx analysis: Phys. Rev. D92, 012010 (2015), arXiv:1504.03634 


\section{inner detector vertex reconstruction}

(2)

ATLAS default tracking algorithm only reconstructs tracks with $d_{0}<10 \mathrm{~mm}$

$\rightarrow$ Modify to allow for high-do tracks

2. Standard primary vertex reconstruction finds vertices consistent $w /$ beam spot $\rightarrow$ Use tracks from step 1, and modify to allow displaced location

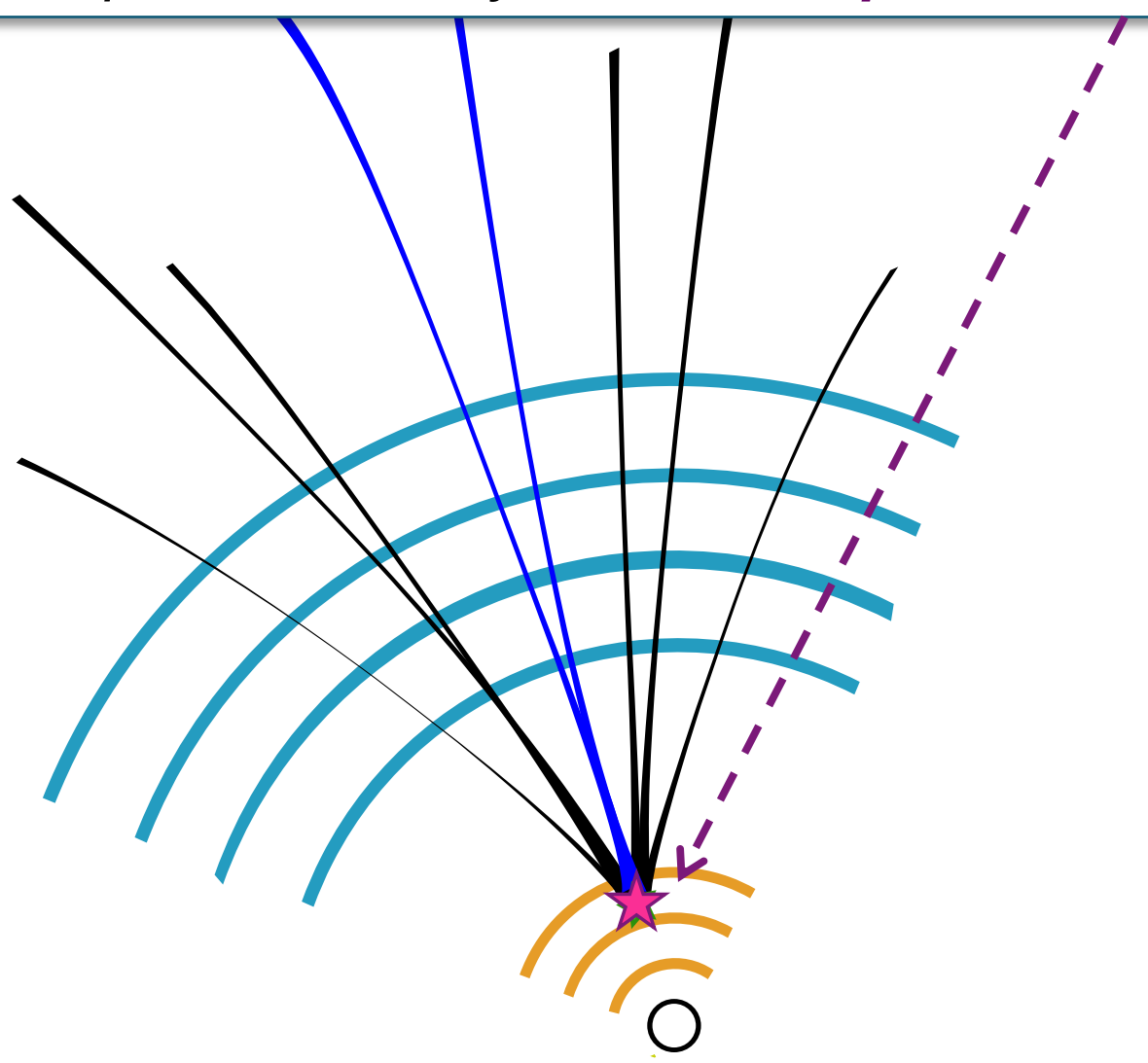

Plots: Phys. Rev. D92, 072004 (2015), arXiv:1504.05162

$2^{\text {nd }}$ IDVx analysis: Phys. Rev. D92, 012010 (2015), arXiv:1504.03634 


\section{inner detector vertex reconstruction}

(2)

ATLAS default tracking algorithm only reconstructs tracks with $d_{0}<10 \mathrm{~mm}$

$\rightarrow$ Modify to allow for high-dol tracks

2. Standard vertex reconstruction finds vertices consistent w/ beam spot

$\rightarrow$ Use tracks from step 1, and modify to allow displaced location

3. No tracks with hits before the vertex

$\rightarrow$ Such tracks are removed, vertex is refit

4. Jet must follow the vertex

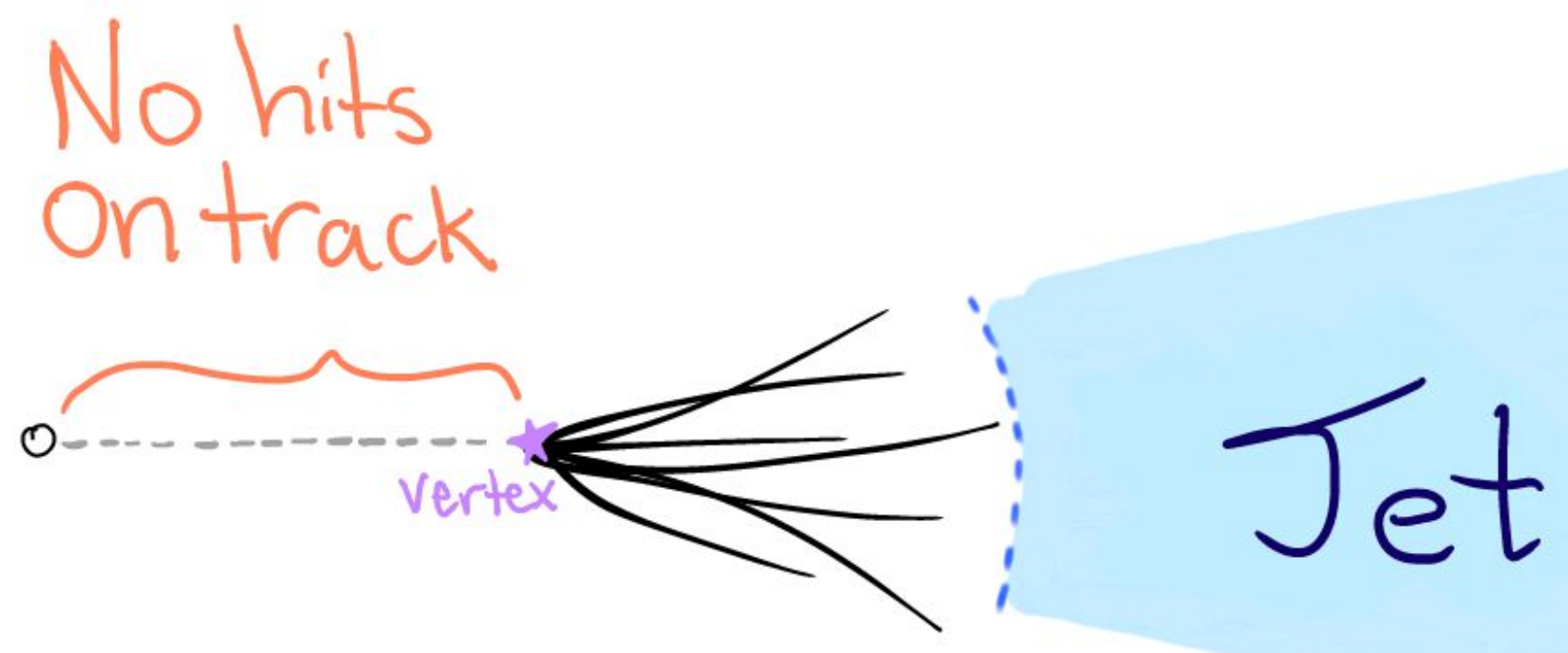

Plots: Phys. Rev. D92, 072004 (2015), arXiv:1504.05162

2nd IDVx analysis: Phys. Rev. D92, 012010 (2015), arXiv:1504.03634 


\section{inner detector vertex reconstruction - efficiency}

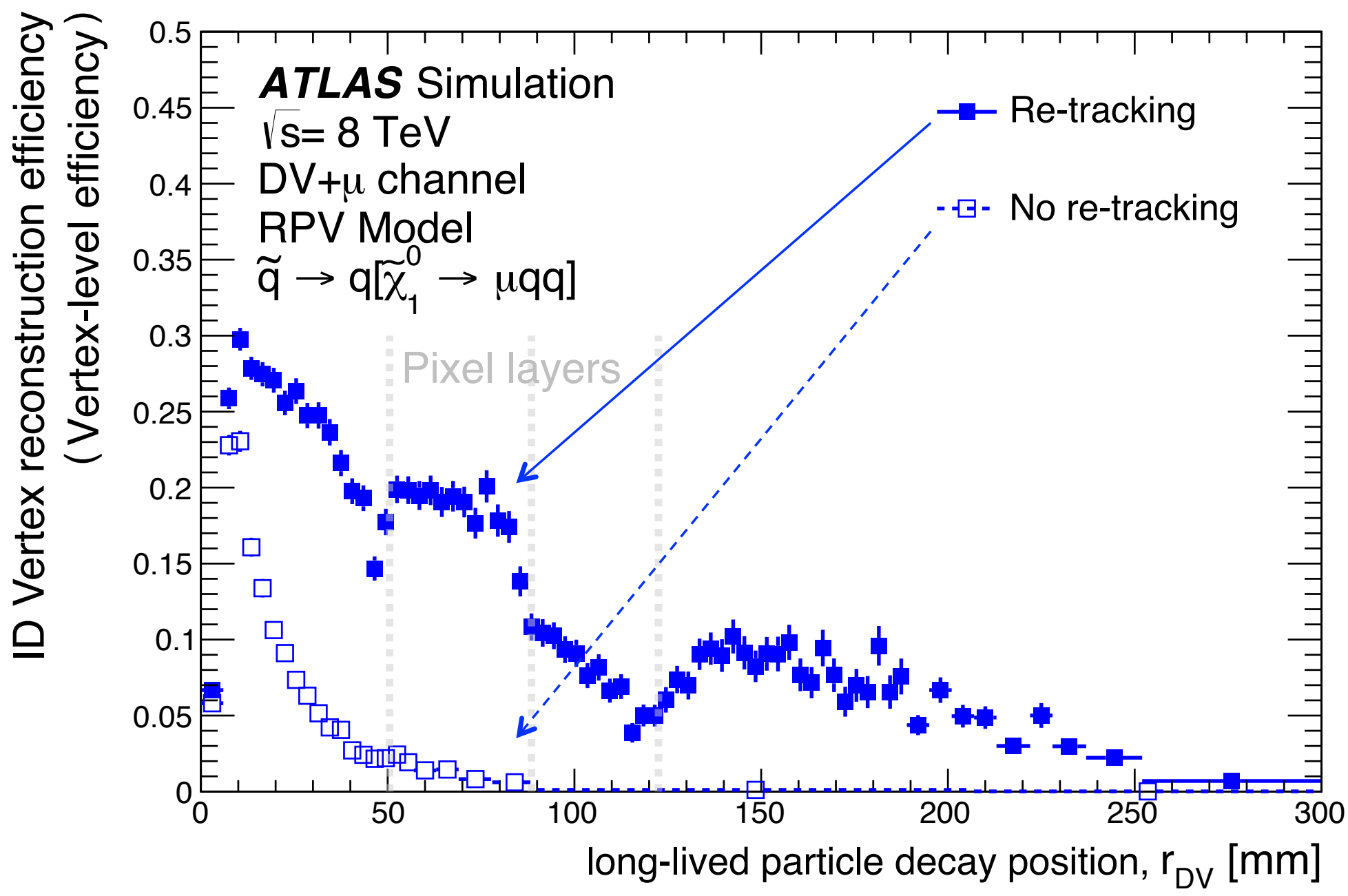

Plots: Phys. Rev. D92, 072004 (2015), arXiv:1504.05162

$2^{\text {nd }}$ IDVx analysis: Phys. Rev. D92, 012010 (2015), arXiv:1504.03634 


\section{hadronic calorimeter decays - definition}

Decays in the HCal result in narrow jets that deposit a small fraction of energy in the ECal and are isolated from ID tracks

1. Dedicated trigger selects low-EMF jets with little track activity

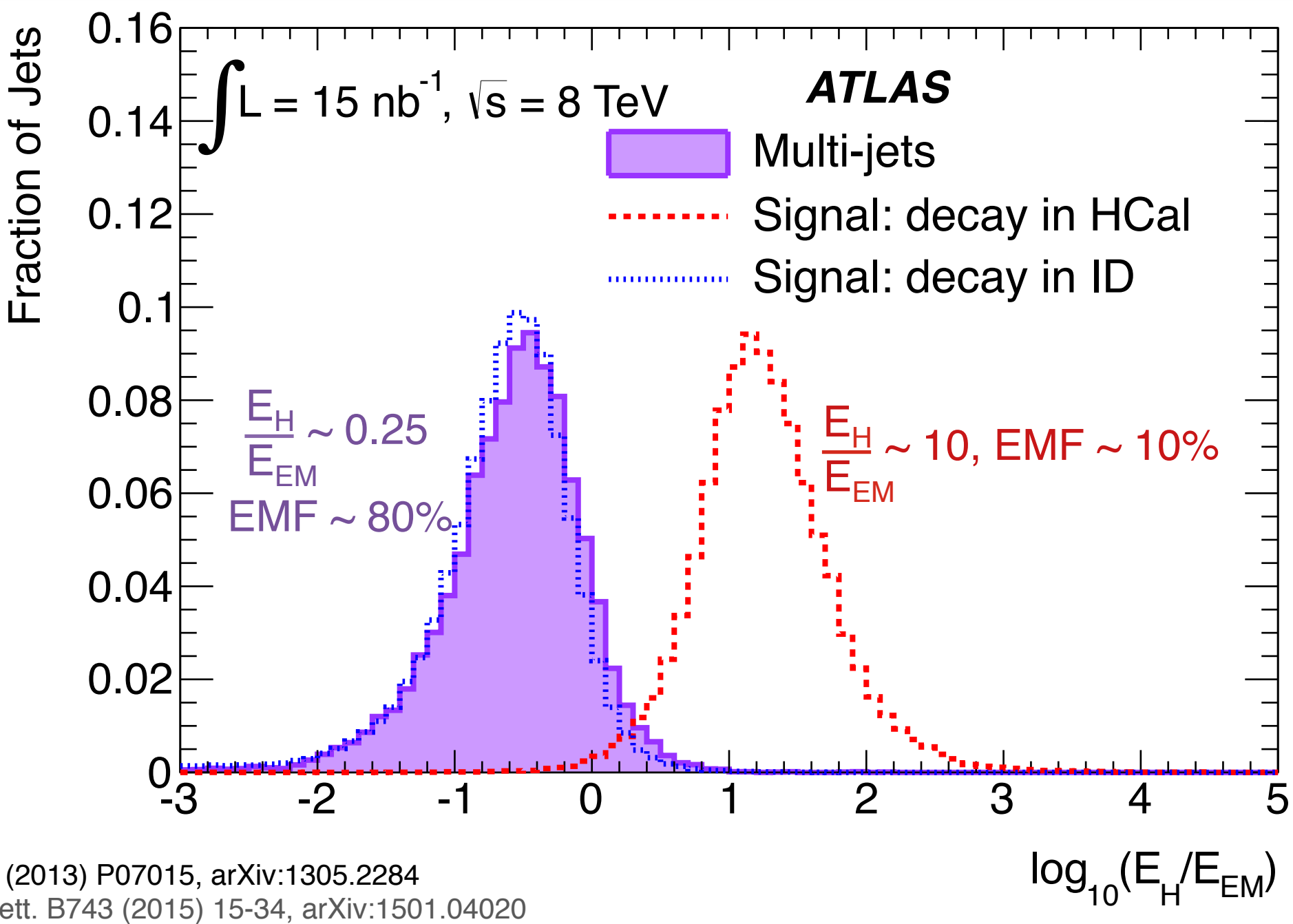




\section{hadronic calorimeter decays - beam halo rejection}

Decays in the $\mathrm{HCal}$ result in narrow jets that deposit a small fraction of energy in the ECal and are isolated from ID tracks

2. Cell and jet timing cuts, muon segment matching to remove beam halo background

Beam halo from muon bremsstrahlung causes low-EMF jets with a skewed timing distribution:
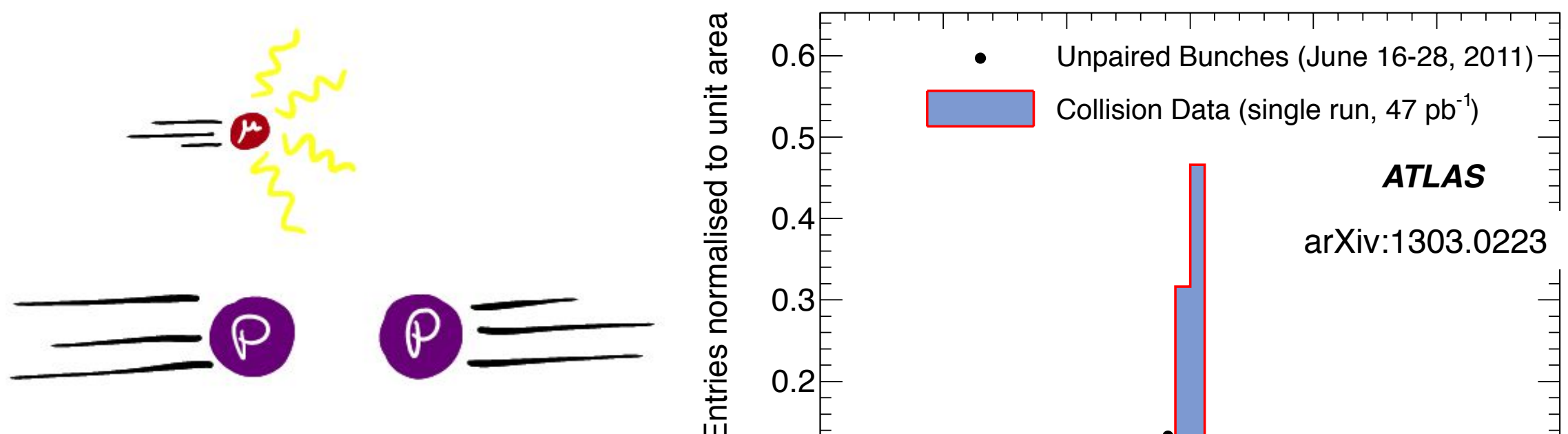

Trigger: JINST 8 (2013) P07015, arXiv:1305.2284 


\section{hadronic calorimeter decays - efficiency}

Decays in the HCal result in narrow jets that deposit a small fraction of energy in the ECal and are isolated from ID tracks

3. Jet energy, track isolation requirements

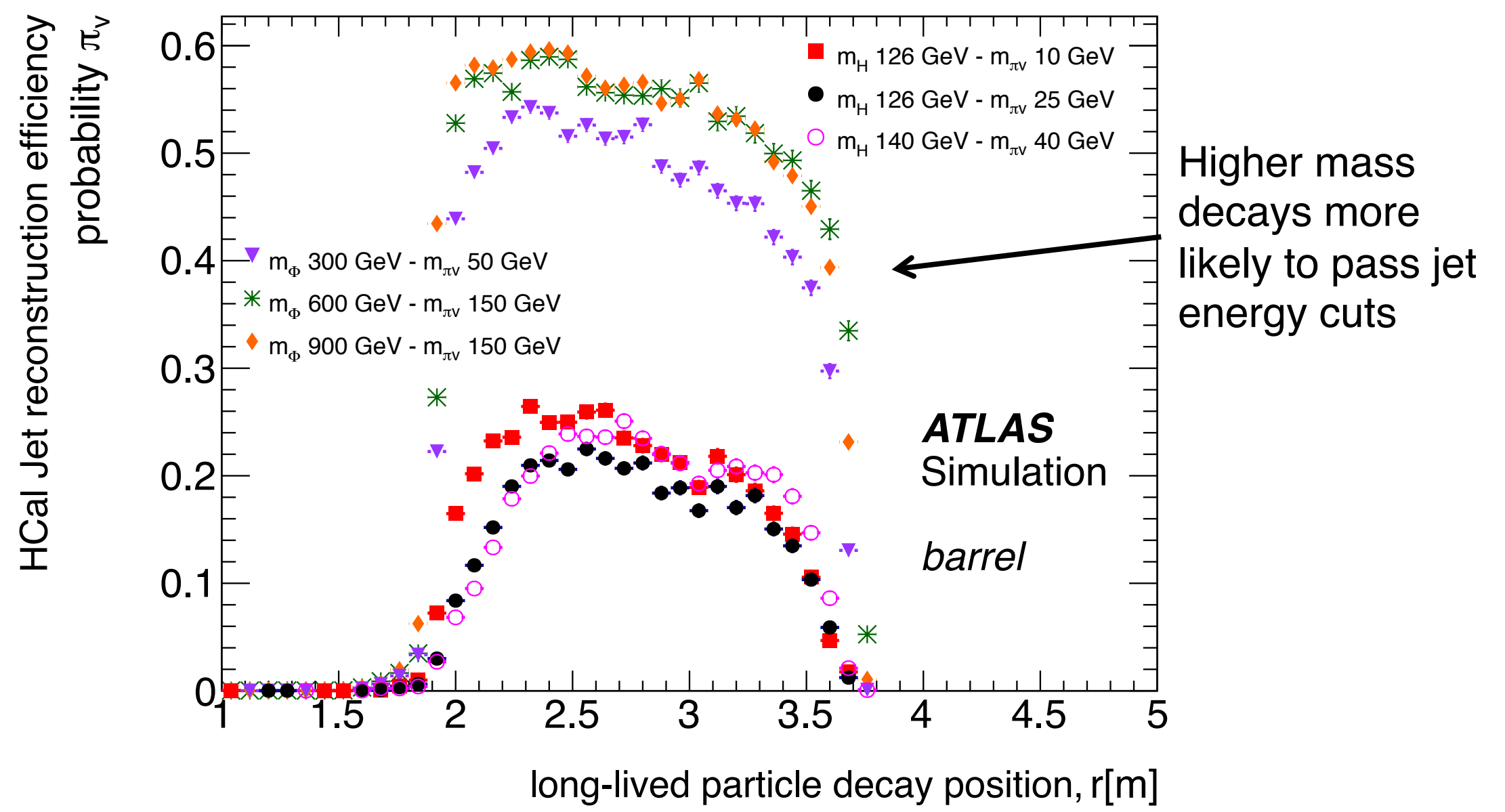

Trigger: JINST 8 (2013) P07015, arXiv:1305.2284 


\section{muon spectrometer decays - triggering}

Muon Rol Cluster trigger selects events with localized clusters of L1Muon trigger objects

$>$ require clusters be isolated from jets, ID tracks
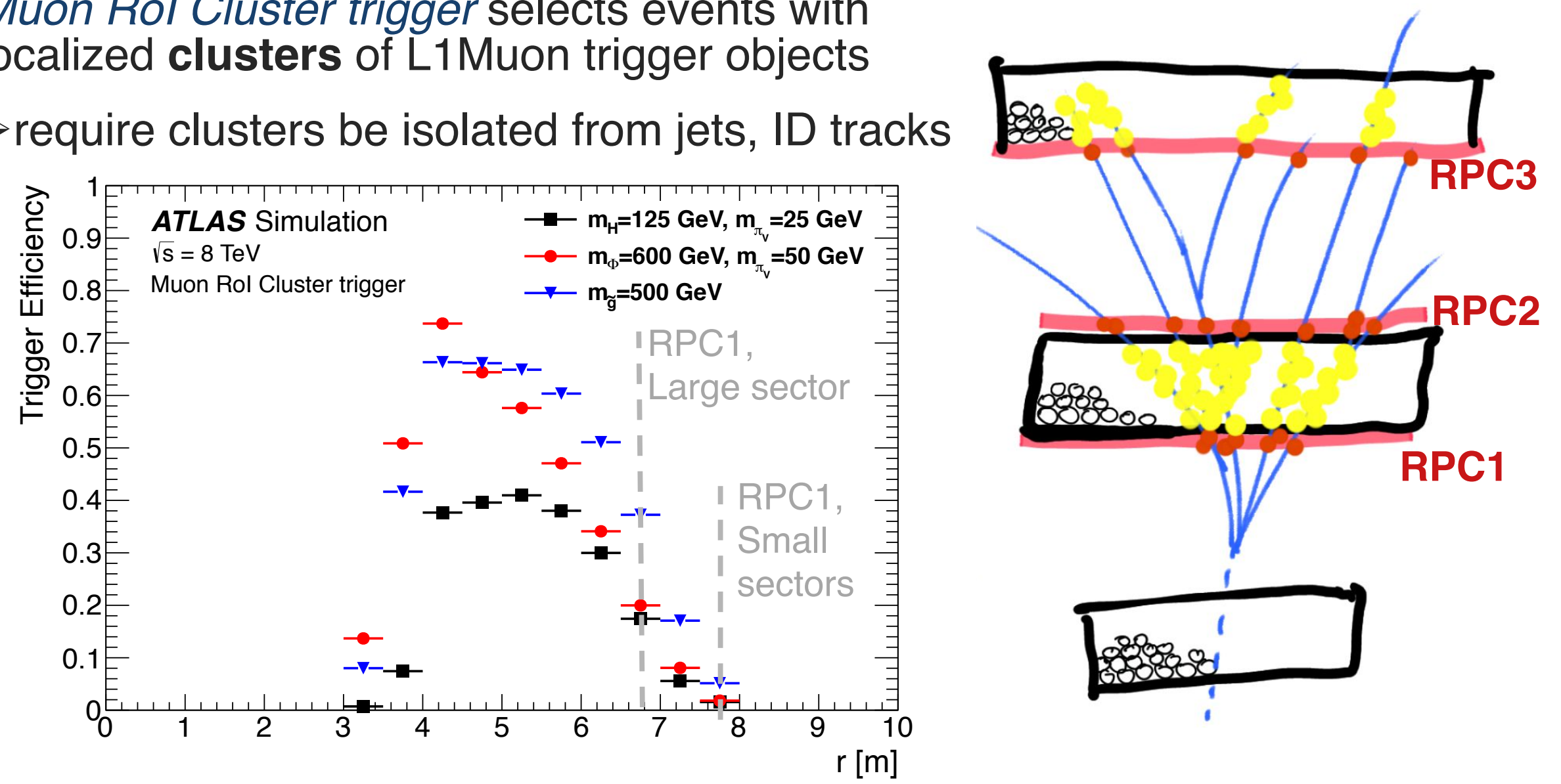

- Efficiency decreases as decay approaches RPC1 $\rightarrow$ jets are too narrow

- No efficiency past RPC1 - need hits there to form L1 Muon trigger objects

- Efficiency in the endcaps is slightly higher

Trigger: JINST 8 (2013) P07015, arXiv:1305.2284

Efficiency: Phys. Rev. D92, 012010 (2015), arXiv:1504.03634

Heather Russell, University of Washington 


\section{muon spectrometer vertex reconstruction}

Purpose-built algorithm reconstructs tracklets and vertices from MDT hits

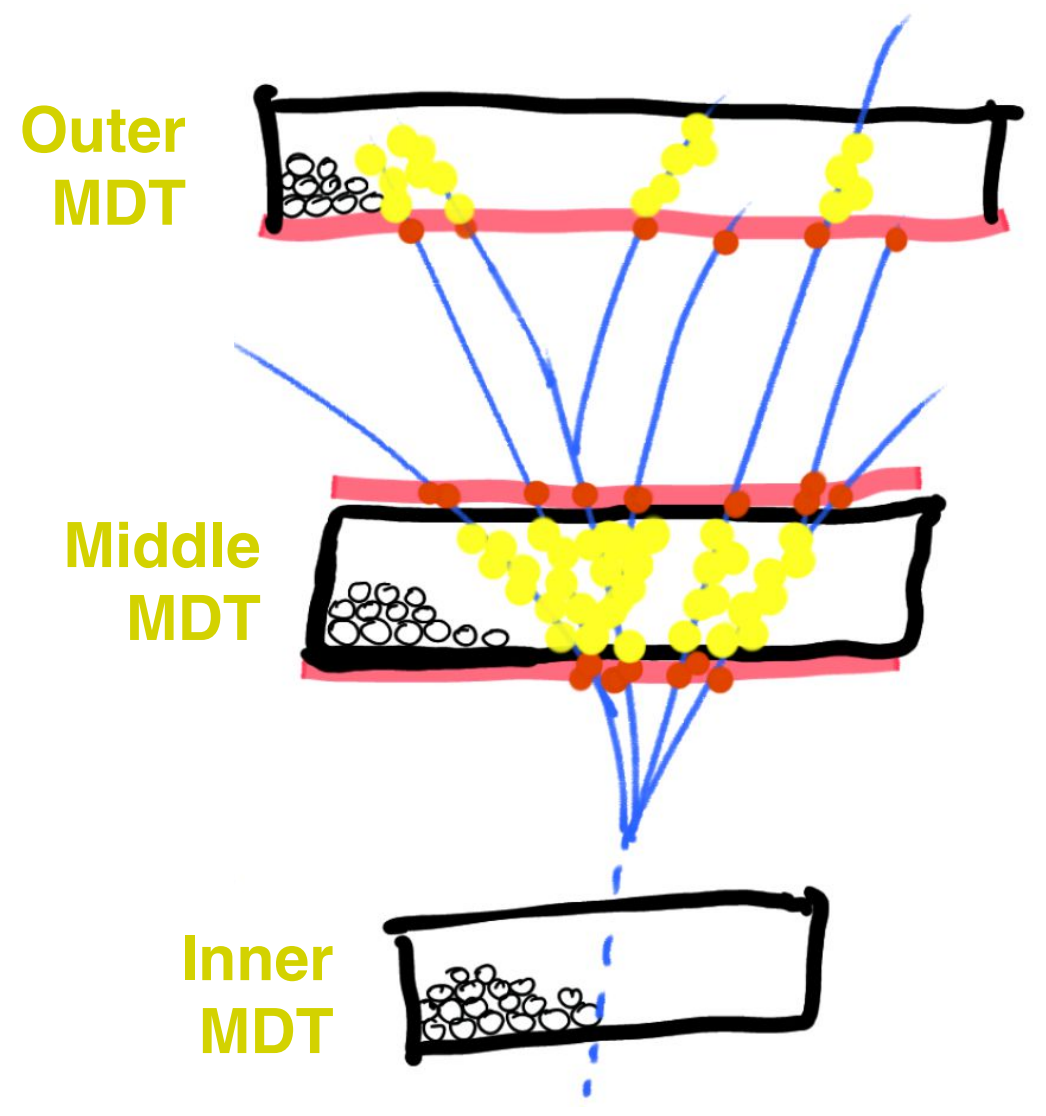

Vx reco: JINST 9 (2014) P02001, arXiv:1311.7070

Efficiency: Phys. Rev. D92, 012010 (2015), arXiv:1504.03634

Heather Russell, University of Washington
Require isolation from tracks and jets to minimize backgrounds from punch-through jets

Efficiency in the barrel as a function of LLP decay position:

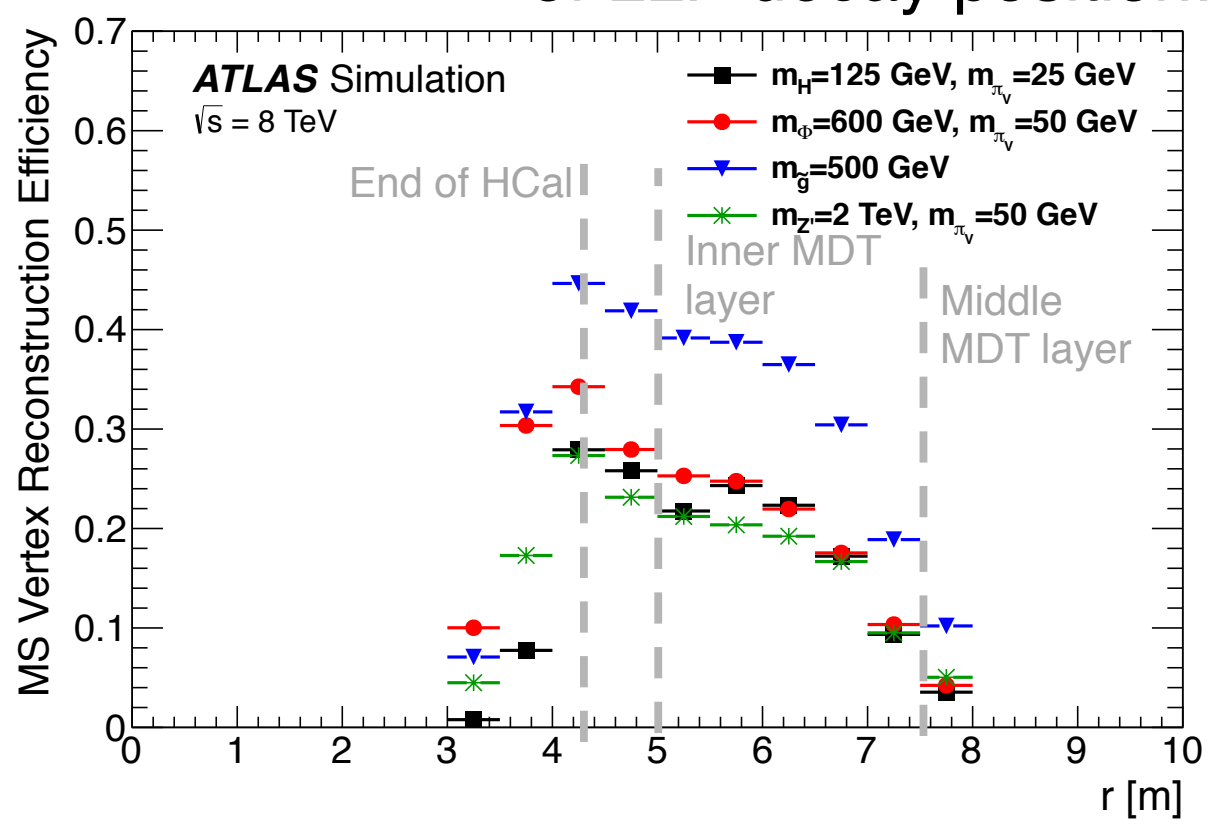

No efficiency much past middle MDT layer $\rightarrow$ need time for decay products to spread out to make enough tracks 


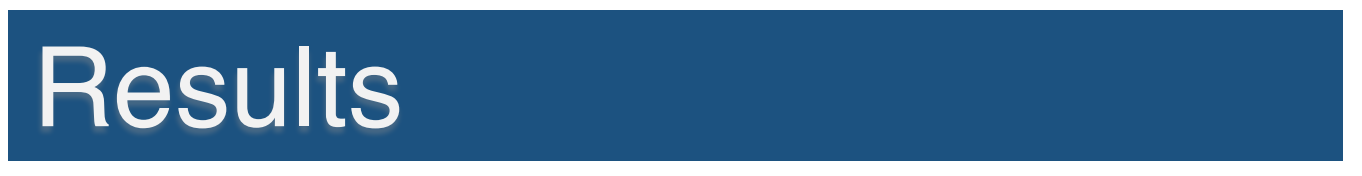




\section{$8 \mathrm{TeV}$ searches with two displaced objects}

\section{Two HCal decays (low-EMF jets)}

2. ID vertex + MS vertex or $2 M S$ vertices*
Phys.Lett. B743 (2015) 15-34, arXiv:1501.04020

Phys. Rev. D92, 012010 (2015) ,arXiv:1504.03634

*one signal model uses a 2 ID vertex signature as well

$>$ Final event selection includes two displaced objects for background reduction

$>$ Example model: Higgs boson decays to longlived scalar pairs

$>$ No excess found in any topology $\rightarrow$ set limits

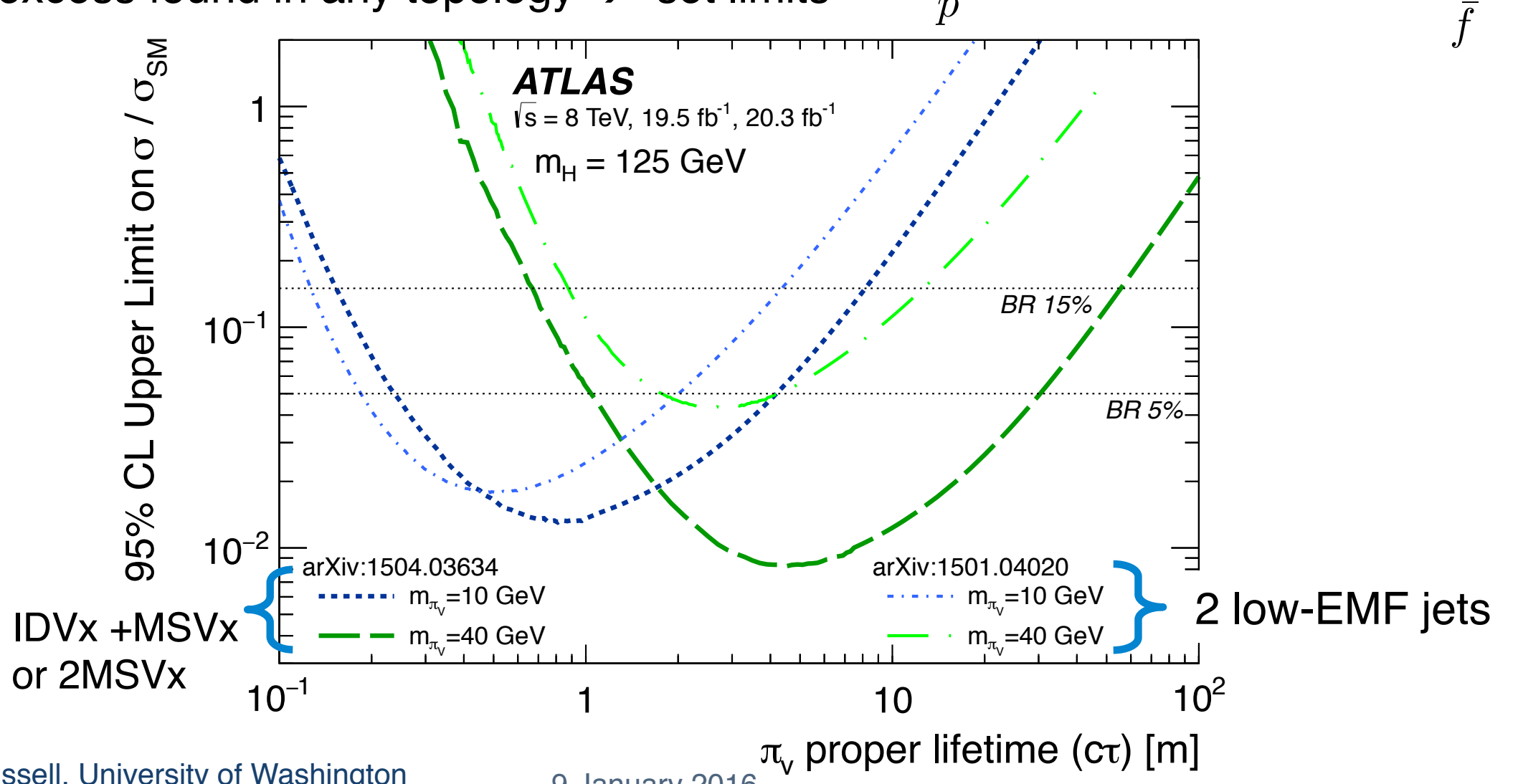

$p$

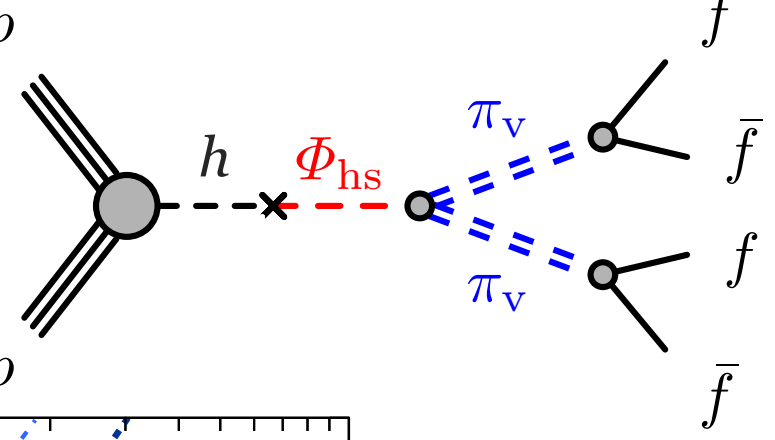




\section{$8 \mathrm{TeV}$ searches with one displaced vertex}

3. ID Vertex + [jets, missing transverse energy, muon, electron]

Phys. Rev. D92, 072004 (2015) arXiv:1504.05162

$>$ No excess found in any topology $\rightarrow$ set limits.

e.g.: Split SUSY model with pair-produced gluinos

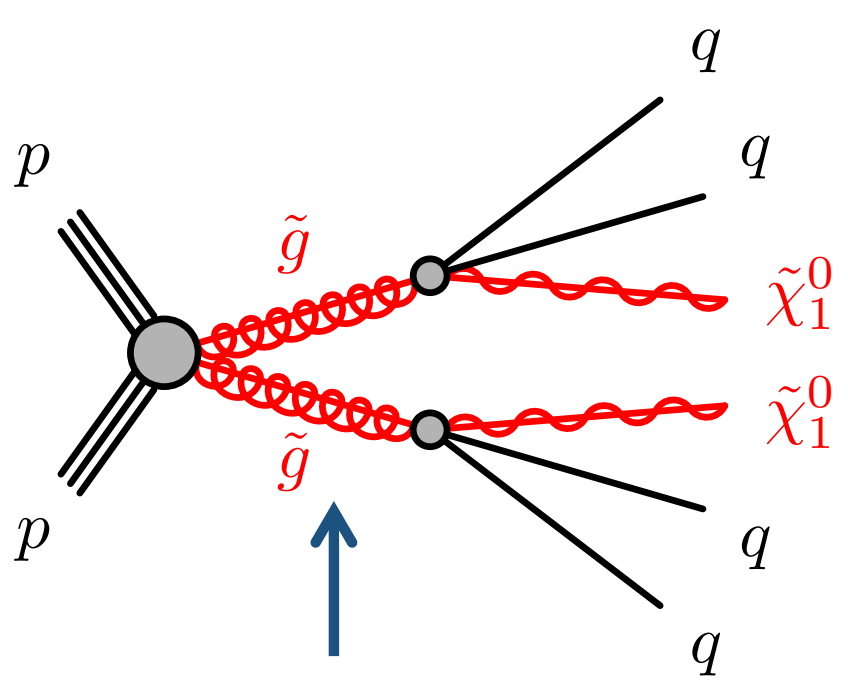

long-lived, neutral gluinos

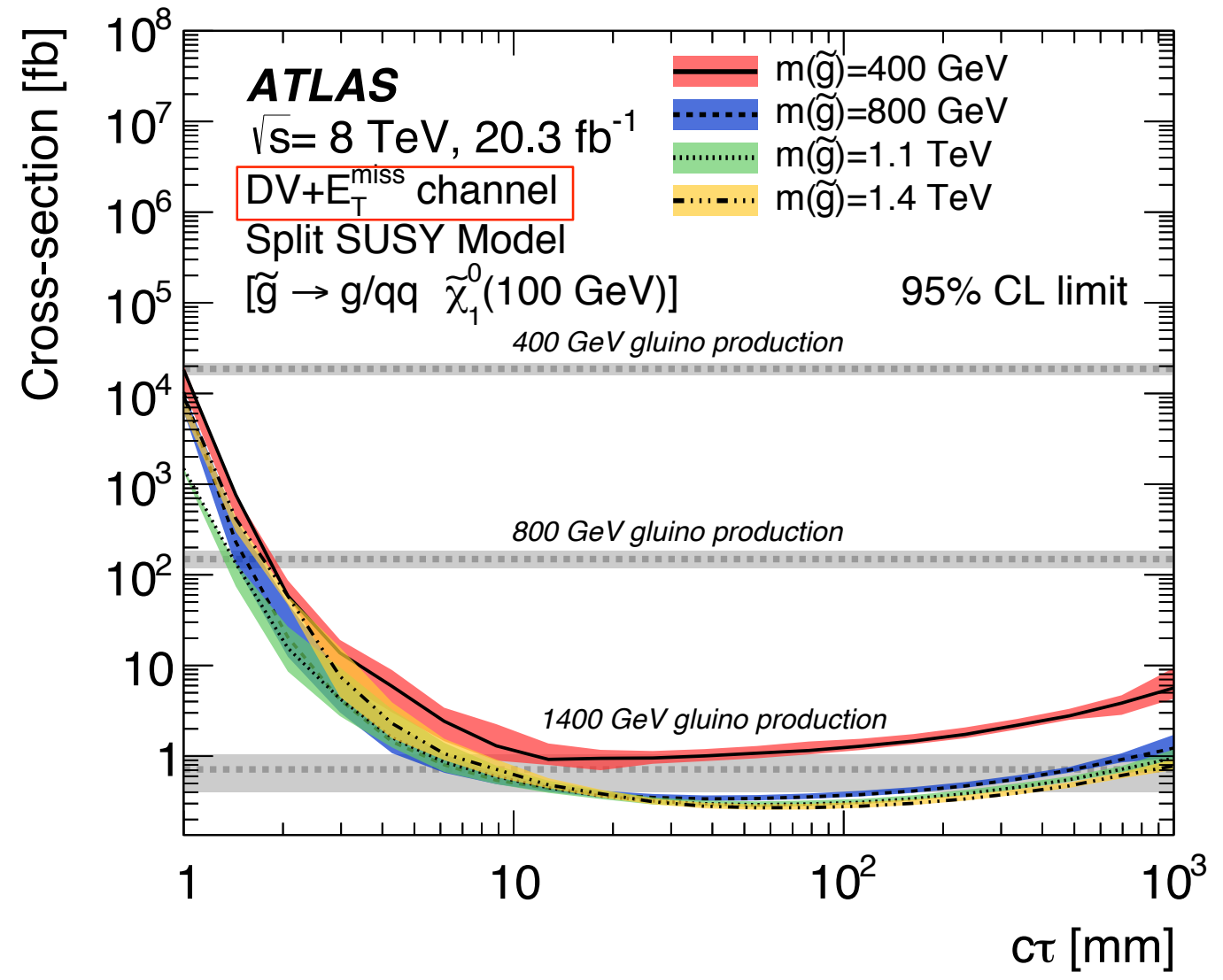




\section{Summary - 1}
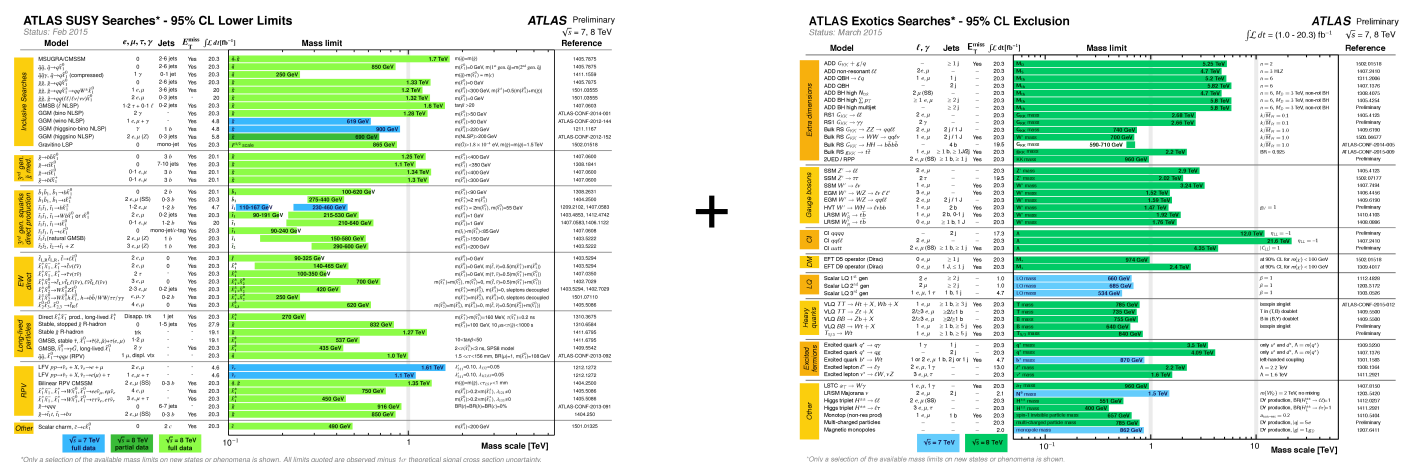

$=$ No new prompt physics.

\section{And, unfortunately,}
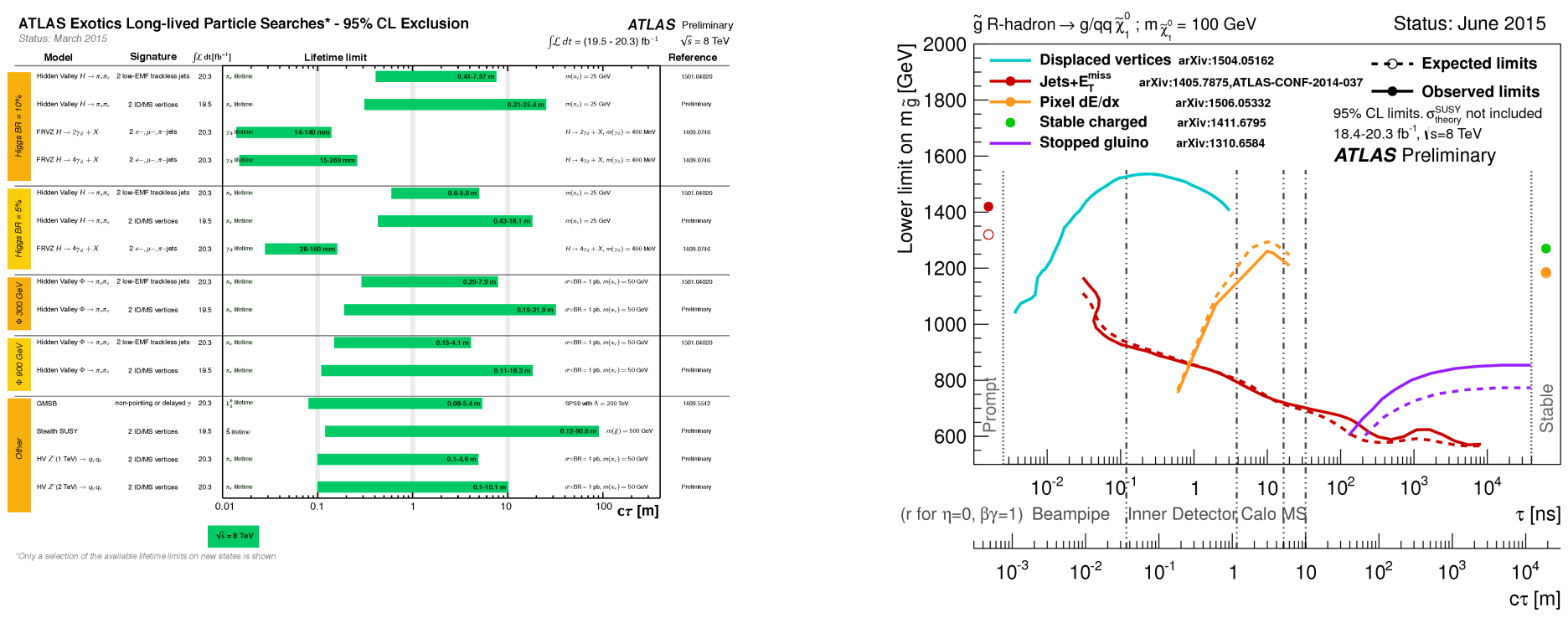

No new displaced physics either.

https://atlas.web.cern.ch/Atlas/GROUPS/PHYSICS/CombinedSummaryPlots/EXOTICS/ https://atlas.web.cern.ch/Atlas/GROUPS/PHYSICS/CombinedSummaryPlots/SUSY/ 


\section{Summary - II}

...however!

$>$ We have novel tools to detect displaced decays in three subsystems: Inner detector, hadronic calorimeter, and muon spectrometer

$>$ Well-defined triggers and reconstruction algorithms for displaced decays

$>$ Results with the 2015 dataset are coming soon!

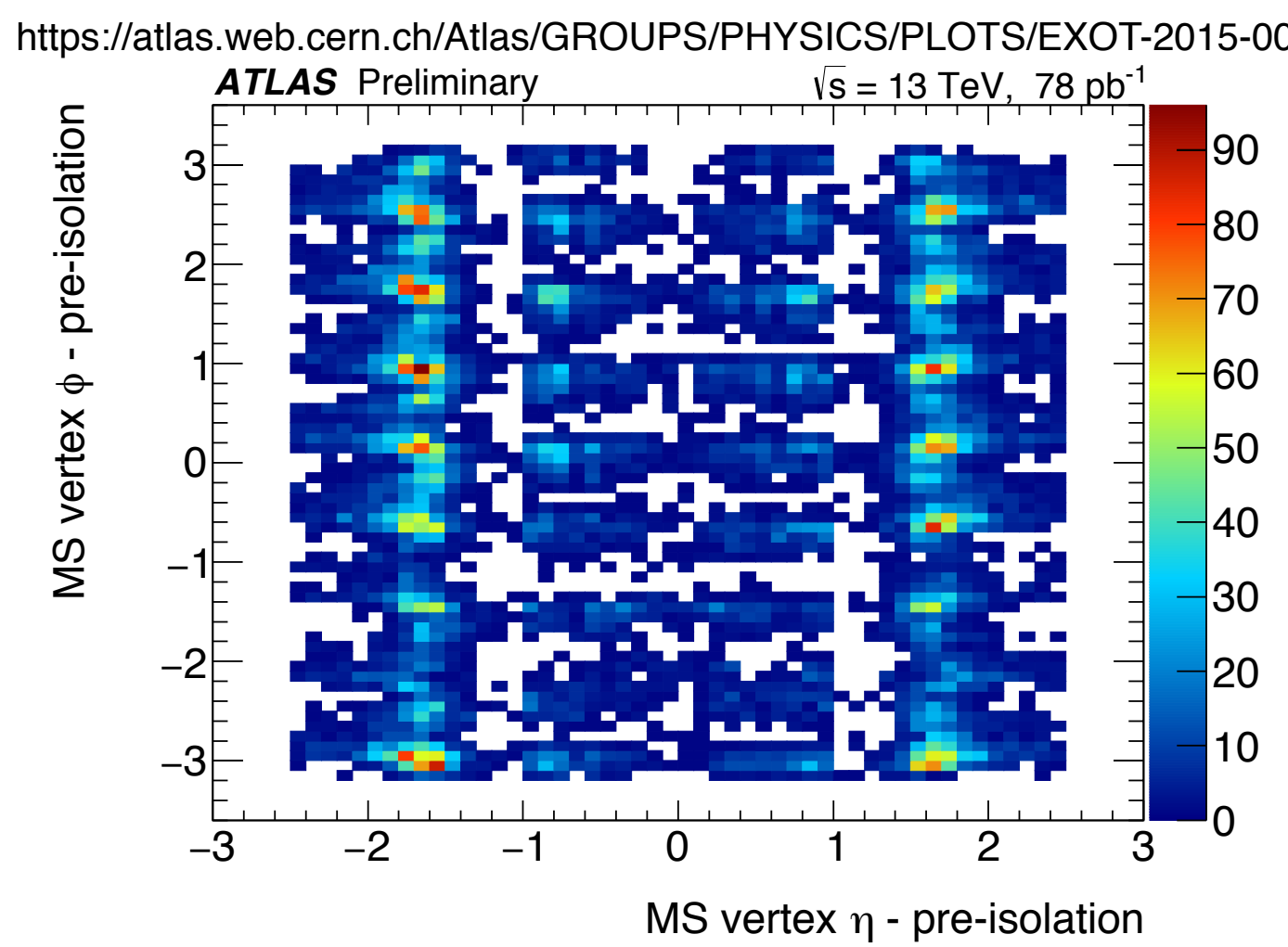


backup material 


\section{References}

Technical papers:

1. Triggers for displaced decays of long-lived neutral particles in the ATLAS detector: JINST 8 (2013) P07015, arXiv:1305.2284, TRIG-2012-02

2. Standalone vertex finding in the ATLAS muon spectrometer: JINST 9 (2014) P02001, arXiv:1311.7070, PERF-2013-01

3. Characterisation and mitigation of beam-induced backgrounds observed in the ATLAS detector during the 2011 proton-proton run:

JINST 8 (2013) P07004, arXiv:1303.0223, DAPR-2012-01

Long-lived particle searches:

1. Search for long-lived neutral particles decaying into lepton jets:

JHEP11(2014)088, arXiv:1409.0746, EXOT-2013-22

2. Search for pair-produced long-lived neutral particles decaying to jets in the ATLAS hadronic calorimeter:

Phys.Lett. B743 (2015) 15-34, arXiv:1501.04020, EXOT-2012-28

3. Search for long-lived, weakly interacting particles that decay to displaced hadronic jets: Phys. Rev. D92, 012010 (2015), arXiv:1504.03634, EXOT-2013-12

4. Search for massive, long-lived particles using multitrack displaced vertices or displaced lepton pairs:

Phys. Rev. D92, 072004 (2015), arXiv:1504.05162, SUSY-2014-02 


\section{$8 \mathrm{TeV}$ searches and sensitivity regions*}

...for hadronically-decaying neutral, long-lived particles (LLPs):

${ }^{\star}$ For decays in the barrel

1. Two decays in the hadronic calorimeter (arXiv:1501.04020, EXOT-2012-28)

2. Two decays in the inner detector or muon spectrometer or one in each (arXiv:1504.03634, EXOT-2013-12)

e.g.:

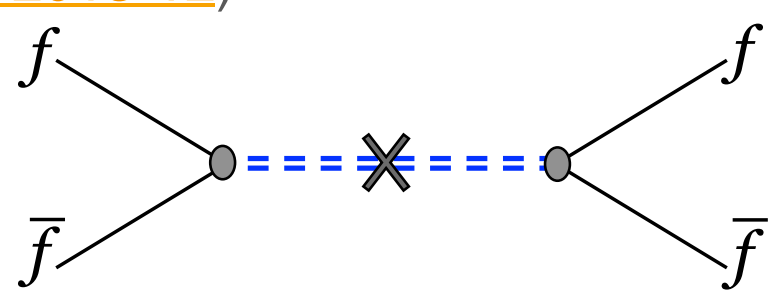

3. One decay in the inner detector + X (arXiv:1504.05162, SUSY-2014-02)

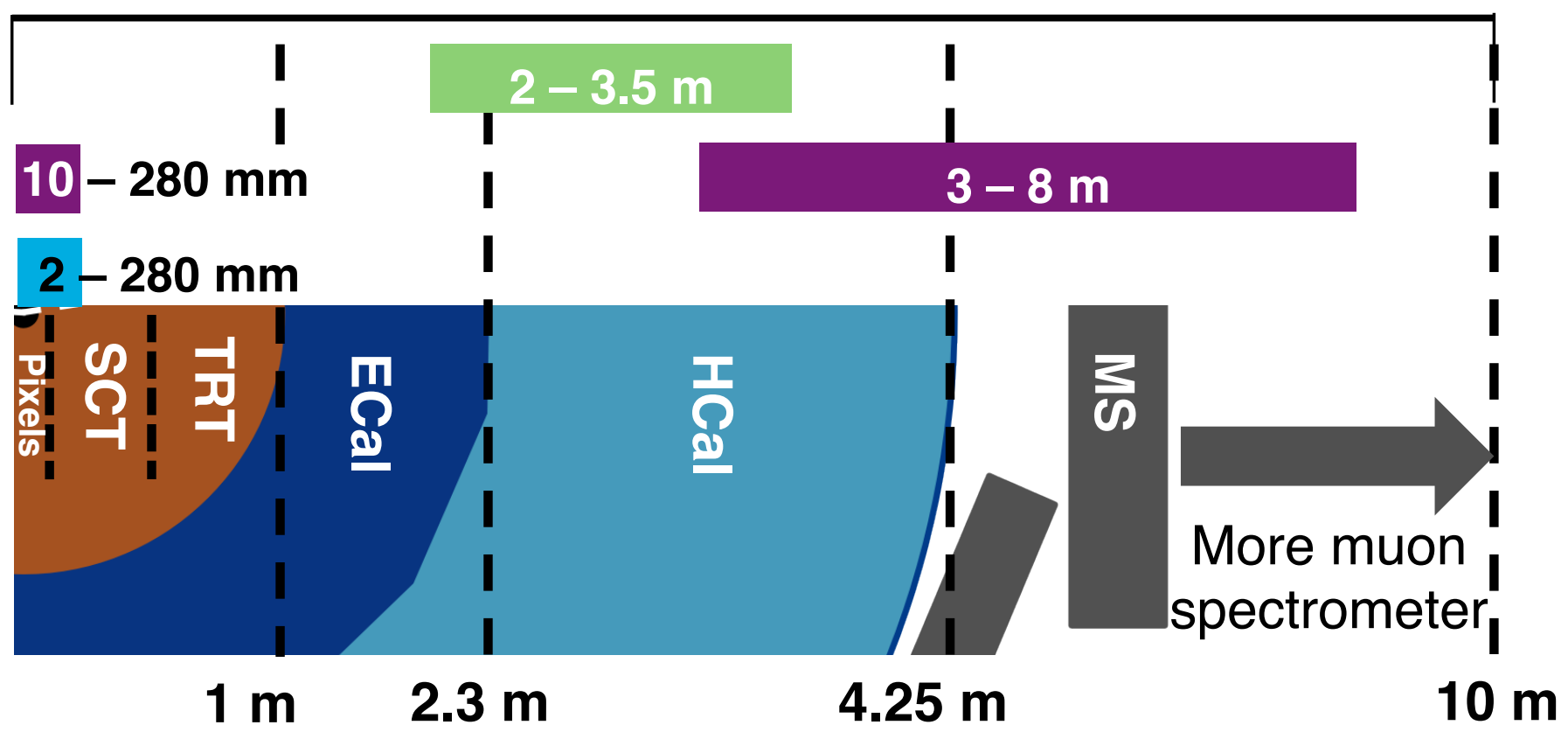




\section{ATLAS Exotics Long-lived Particle Searches* - 95\% CL Exclusion}

Status: March 2015 https://atlas.web.cern.ch/Atlas/GROUPS/PHYSICS/CombinedSummaryPlots/EXOTICS/

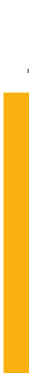

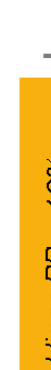

Model

Signature $\quad \int \mathcal{L} \mathrm{dt}\left[\mathrm{fb}^{-1}\right]$

Hidden Valley $H \rightarrow \pi_{\mathrm{\vee}} \pi_{\mathrm{V}} \quad 2$ low-EMF trackless jets 20.3

\begin{tabular}{lll|l} 
Hidden Valley $H \rightarrow \pi_{\mathrm{v}} \pi_{\mathrm{V}}$ & 2 ID/MS vertices & 19.5 & $\pi_{\mathrm{v}}$ lifetime
\end{tabular}

FRVZ $H \rightarrow 2 \gamma_{d}+X \quad 2 e^{-,}, \mu-, \pi$-jets

$\mathrm{FRVZ} H \rightarrow 4 \gamma_{d}+X$

$2 e-, \mu-, \pi-$ jets $\quad 20.3$

20.3

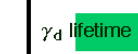

Lifetime limit

Hidden Valley $H \rightarrow \pi_{\mathrm{v}} \pi_{\mathrm{V}} \quad 2$ low-EMF trackless jets 20.3

Hidden Valley $H \rightarrow \pi_{\mathrm{v}} \pi_{\mathrm{v}} \quad 2$ ID/MS vertices

\begin{tabular}{ll|l} 
& 19.5 & $\pi_{\mathrm{v}}$ lifetime
\end{tabular}

$\mathrm{FRVZ} H \rightarrow 4 \gamma_{d}+X$

$2 e-, \mu-, \pi$-jets

20.3

$\gamma_{d}$ lifetime

$28-160 \mathrm{~mm}$

Hidden Valley $\Phi \rightarrow \pi_{\mathrm{v}} \pi_{\mathrm{v}} \quad 2$ low-EMF trackless jets $\quad 20.3$

Hidden Valley $\Phi \rightarrow \pi_{\mathrm{v}} \pi_{\mathrm{V}}$

2 ID/MS vertices

$19.5 \quad \pi_{\mathrm{v}}$ lifetime

$\pi_{\mathrm{y}}$ lifetime
ATLAS Preliminary $\int \mathcal{L} d t=(19.5-20.3) \mathrm{fb}^{-1} \quad \sqrt{s}=8 \mathrm{TeV}$

Reference

1501.04020

Preliminary

1409.0746

$H \rightarrow 2 \gamma_{d}+X, m\left(\gamma_{d}\right)=400 \mathrm{MeV}$

$H \rightarrow 4 \gamma_{d}+X, m\left(\gamma_{d}\right)=400 \mathrm{MeV}$

1409.0746

1501.04020

Preliminary

1409.0746

1501.04020

Preliminary

1501.04020

Preliminary

1409.5542

Preliminary

Preliminary

Preliminary

$\sigma \times \mathrm{BR}=1 \mathrm{pb}, m\left(\pi_{\mathrm{v}}\right)=50 \mathrm{GeV}$

$\mathrm{HV} Z^{\prime}(2 \mathrm{TeV}) \rightarrow q_{\vee} q_{V} \quad 2$ ID/MS vertices

20.3

0.1-10.1 m ${ }^{100} \mathrm{c} \tau[\mathrm{m}]$ 


\section{Other SUSY long-lived searches}

ID Vertex + [jets, MET, muon, electron]

- Jet+MET search, reinterpreted for metastable gluinos

\section{Metastable charged} particles with large ionisation energy loss

Out-of-time gluino decays

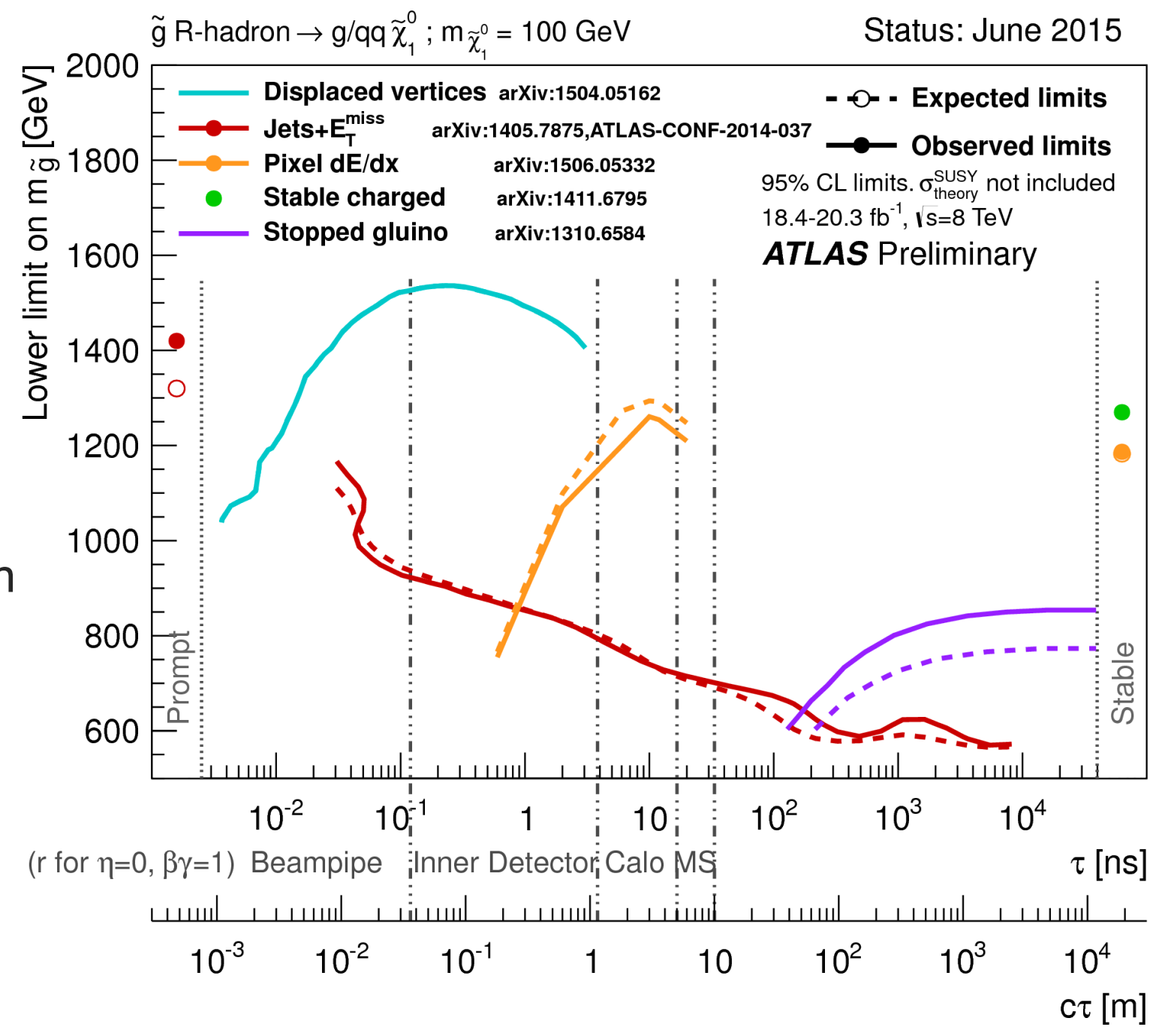

https://atlas.web.cern.ch/Atlas/GROUPS/PHYSICS/CombinedSummaryPlots/SUSY/ 


\section{Aside - hidden sectors}

Sets of particles + interactions that are scalars under the standard model gauge groups.

Without additional phenomenology, hidden sector particles:

- Won't be produced by standard model particles

- Won't decay to standard model particles

- Won't have any interactions with standard model particles and our detectors are all made out of standard model particles!

They are... hidden.
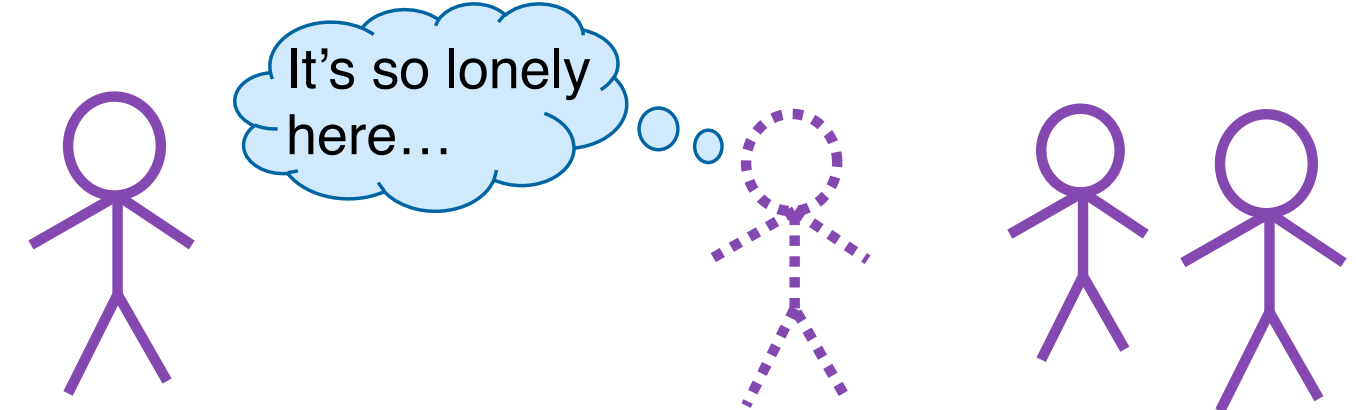

For hidden sector particles to be produced or decay, need some coupling between hidden sector and standard model

small couplings $=$ long lifetimes! 


\section{inner detector geometry 1}

- $2 \mathrm{~T}$ solenoidal magnetic field (along z) allows for charge/ momentum measurements

- Pixels and silicon microstrip trackers (SCTs) allow for precise tracking measurements

Barrel resolution:

-Pixels:

$10 \mu \mathrm{m}$ in $(R-\varphi), 115 \mu \mathrm{m}$ in $z$ -SCT:

$17 \mu \mathrm{m}$ in $(R-\varphi), 580 \mu \mathrm{m}$ in $z$

- Good (transverse) resolution allows for precise tracking + vertex reconstruction

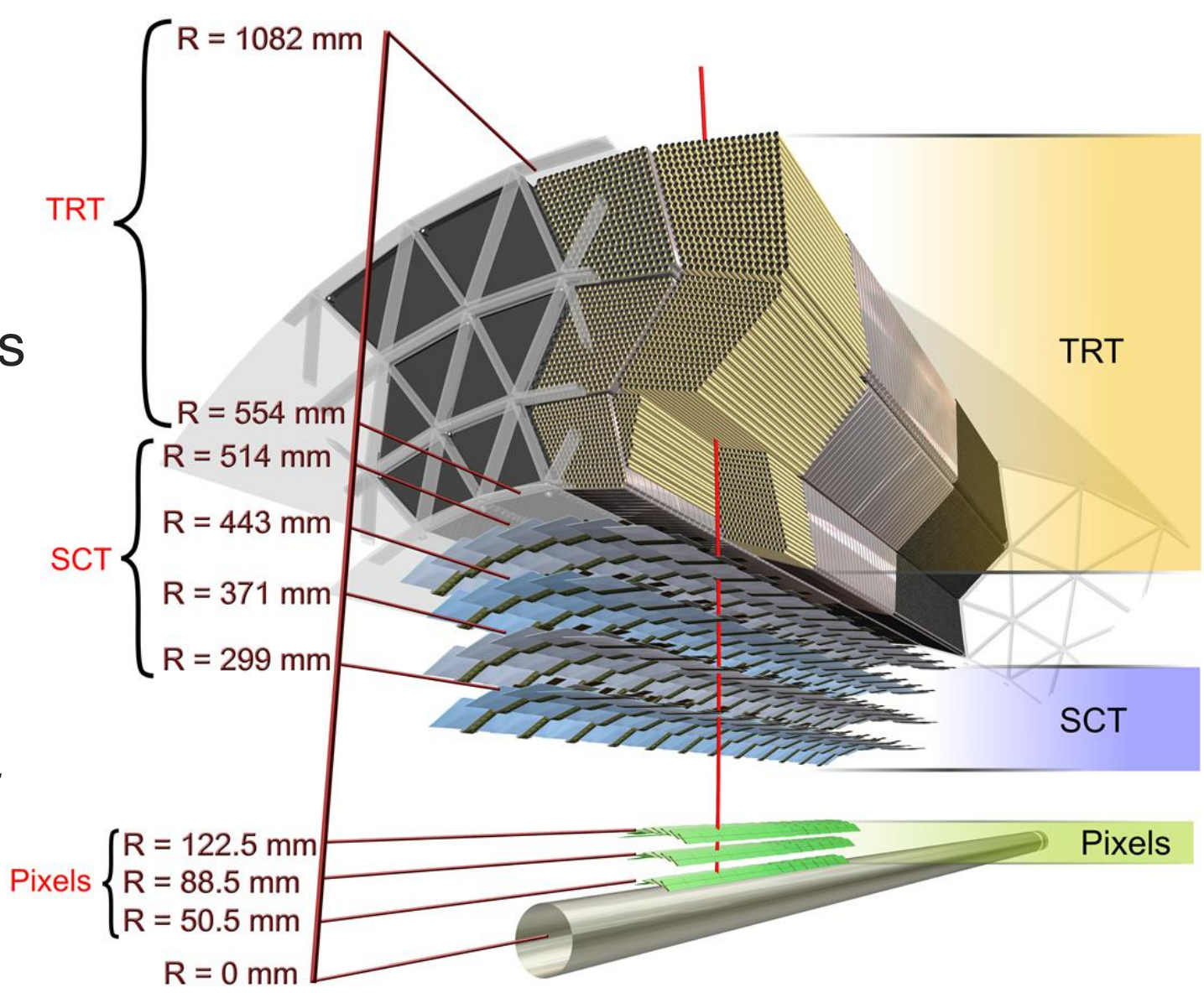

${ }^{1}$ Expected Performance of the ATLAS Experiment: arXiv:0901.0512v4 


\section{inner detector vertex reconstruction}

$(2)$ ATLAS

\section{Remove all vertices consistent with hadronic material interactions}

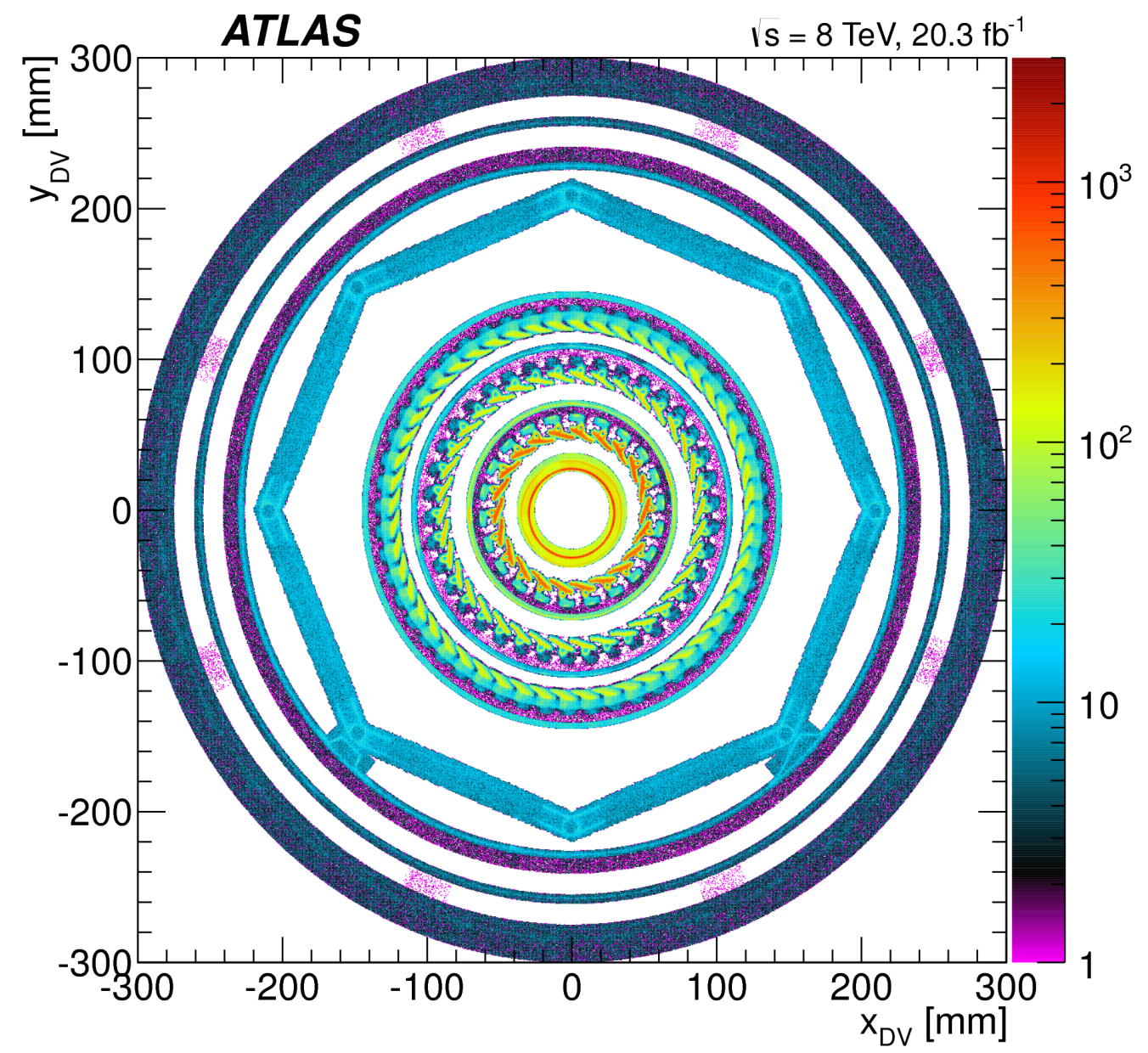

Density of $<5$-track vertices reconstructed within the inner detector material

Plots: Phys. Rev. D92, 072004 (2015), arXiv:1504.05162

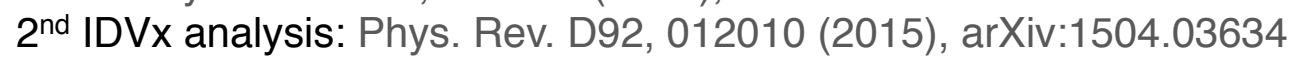




\section{ID vertex reconstruction efficiency}

Phys. Rev. D92, 012010 (2015), arXiv:1504.03634

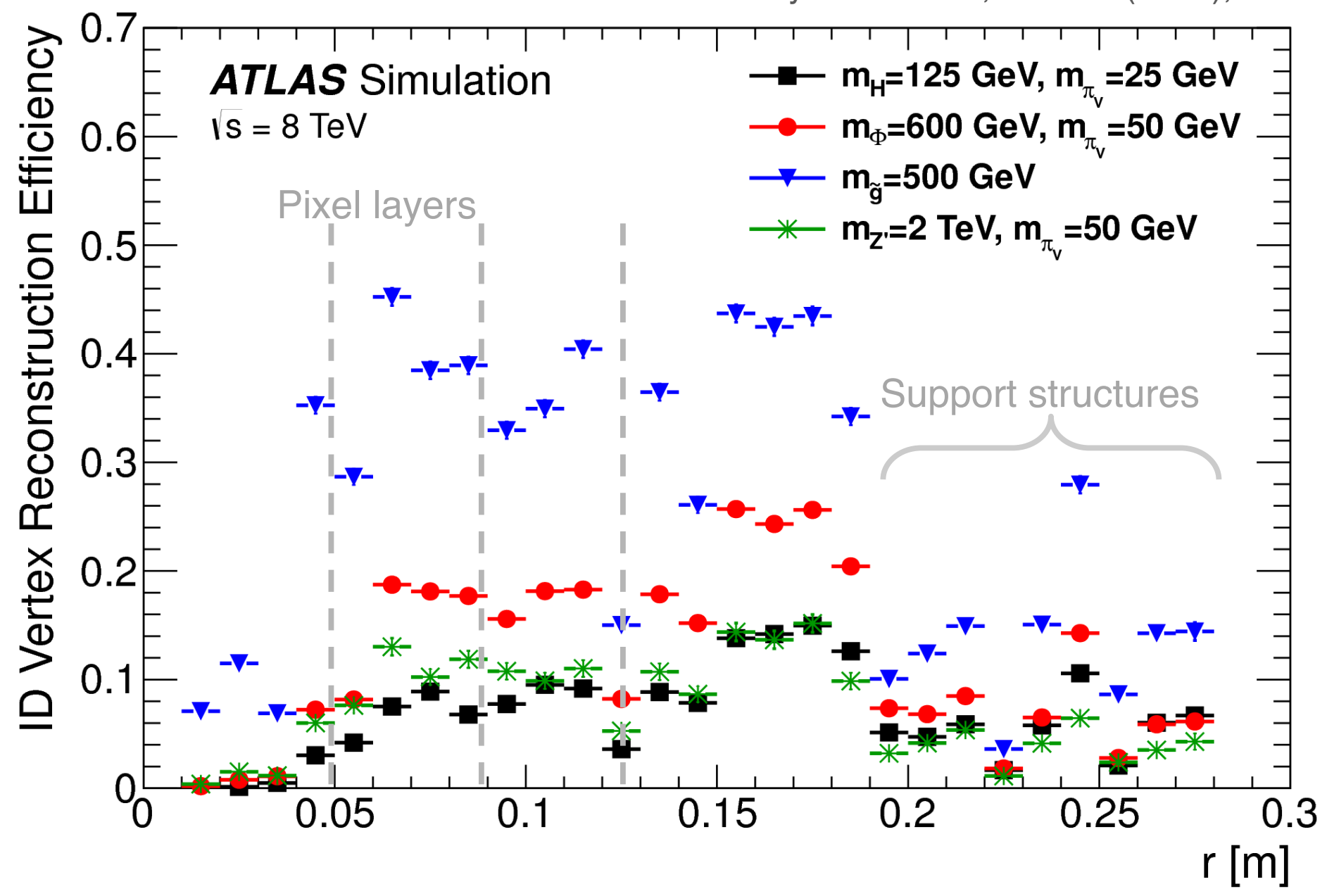

Jaggedness is caused by the application of the material veto - lower efficiencies correlate to a radius where there is material present 


\section{ID vertex reconstruction - quality criteria details}

Determine a set of good vertex criteria (GVC) to minimize number of background vertices (from multi-jet events)

\begin{tabular}{cc|c} 
Background vertices & Signal vertices & Criteria* $^{*}$ \\
\hline $\begin{array}{c}\text { Low track-multiplicities } \\
(<6 \text { tracks/vertex })\end{array}$ & Higher track-multiplicities & nTracks $>5(7)$ \\
$\begin{array}{c}\text { Vertices not necessarily } \\
\text { followed by a jet }\end{array}$ & $\begin{array}{c}\text { Vertices should be followed } \\
\text { by a jet }\end{array}$ & $\mathrm{dR}(\mathrm{vtx}, \mathrm{jet})<0.4(0.6)$ \\
$\begin{array}{c}\text { Can be from random } \\
\text { tracks crossing a low- } \\
\text { track multiplicity } \\
\text { secondary vertex }\end{array}$ & All tracks come from same & $X^{2}$ prob. of fit $>0.001$ \\
& vertex &
\end{tabular}

*(values in parentheses are for Hidden Valley Z' signal benchmarks - a model with a busier final state than just two LLPs) 


\section{muon spectrometer - geometry}

JINST 9 (2014) P02001, arXiv:1311.7070

- Air-core toroid magnetic field $0.5 \mathrm{~T}$ in barrel, $1 \mathrm{~T}$ in endcaps, symmetric in $\varphi$

- Resistive Plate Chambers (RPCs) used for triggering, provide $\varphi$ information for MDT hits (TGCs in endcaps)

- Muon drift tubes (MDTs) used for vertex finding.

- Avg. resolution: $35 \mu \mathrm{m} /$ chamber, limiting factor is tube resolution

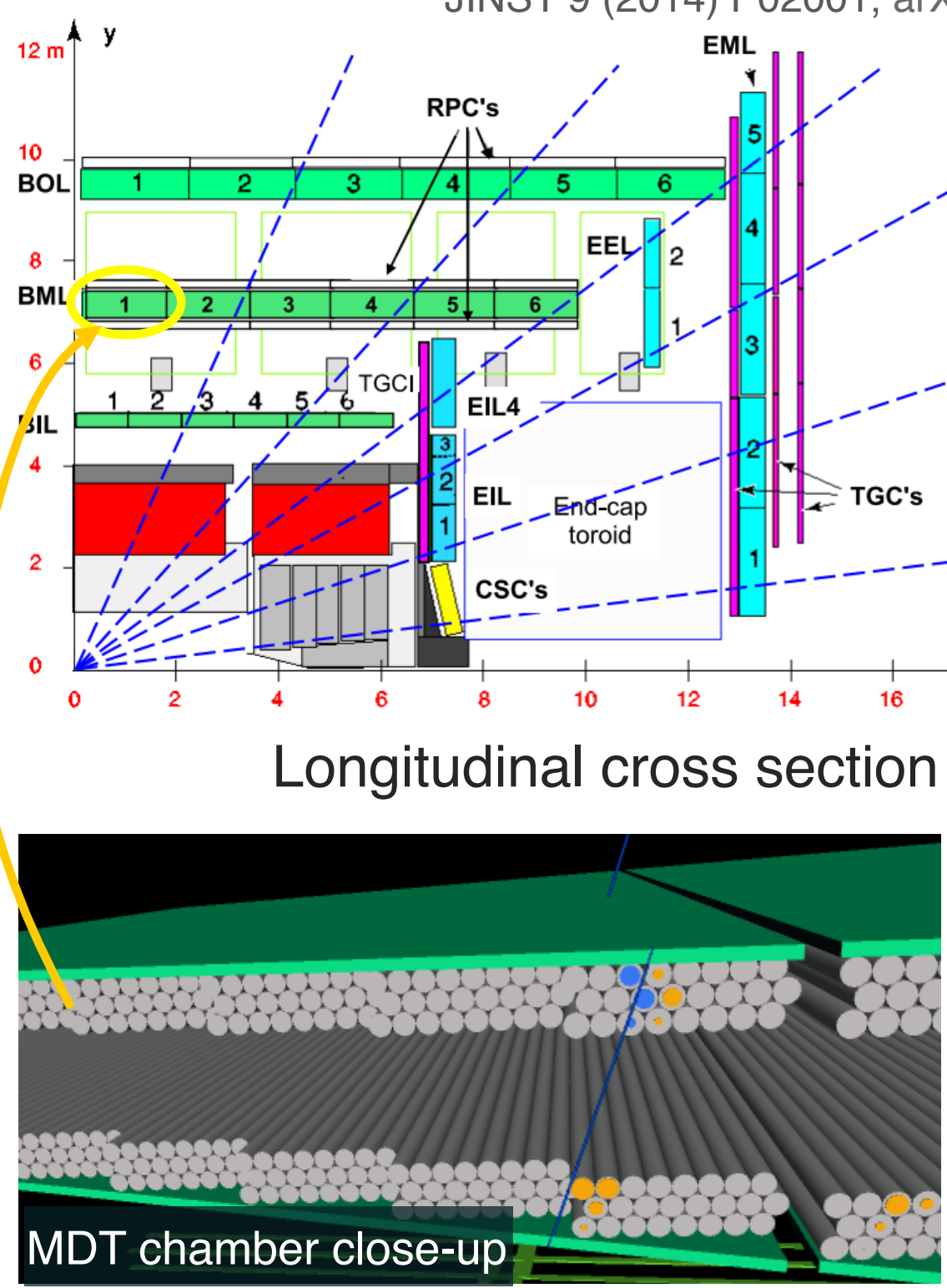

